\title{
Computing Technology: Special Announcement MacPsych: An electronic discussion list and archive for psychology concerning the Macintosh computer
}

\author{
CHARLES HUFF \\ St. Olaf College, Northfield, Minnesota \\ and \\ BLAKE SOBILOFF \\ University of Maryland, College Park, Maryland
}

\begin{abstract}
MacPsych is an electronic mail discussion list and an electronic archive for those using the Macintosh in psychology. The discussion list allows psychologists from around the world to ask questions and discuss issues relating to the use of the Macintosh in psychology. The archive is a place where list members may share software and other files of relevance. The Society for Computers in Psychology sponsors the discussion list, and the journal Behavior Research Methods, Instruments, \& Computers has designated the archive as the distribution site for Macintosh software and files described in its pages. Those interested may join the list by sending electronic mail to macpsych-request@stolaf.edu. The archive can be accessed via anonymous ftp at ftp.stolaf.edu in the directory pub/macpsych.
\end{abstract}

At a meeting of the Macintosh user group at the Society for Computers in Psychology (SCiP) in November 1990 , several members complained that the only time they were able to share technical problems and solutions came at the annual meeting. For people interested in computing, it was an easy step from this complaint to the establishment of an electronic discussion list that would allow them to talk about these issues over electronic mail.

The list was established in December of 1990 at St. Olaf College, with the electronic mail address of macpsych@ stolaf.edu. The first list members came from the small group of users at that meeting. We expected the list would be a place for discussion among people concerned about minor technical issues with the Macintosh computer and psychological experimentation.

We were surprised by the response from those who heard of the group and signed up to participate. MacPsych now has over 330 members from North America, Europe, Australia, and New Zealand. In discussions on the list, we have considered how best to do millisecond timing, how to display complex images quickly on the screen, the use of soft-

Thanks to Dick Lehman for his generous help with the establishment of MacPsych. Thanks to Craig Rice for writing and documenting the program ftpbymail. Thanks to Doug Chute, The Society for Computers in Psychology, and Behavior Research Methods, Instruments, \& Computers for their support and for recognizing the importance of electronic communication. Stuffit Classic (Aladdin Systems, Inc.) is a shareware product-please pay the shareware fee if you choose to use it, or use only UnStuffit Classic. Any comments or requests should be sent to C. Huff, Psychology Department, St. Olaf College, Northfield, MN 55057 , or to macpsych-request@stolaf.edu. ware in the classroom, and other issues of relevance to psychologists using Macintosh computers.

By popular demand, an electronic archive has been added that allows software and documents that relate to psychology and the Macintosh to be shared. This archive currently contains software and documents that some list members have written and software that others have written (e.g., utilities) that might be helpful to the list community. There are, for example, files of source code for quick screen display of images, schematics of hardware for doing millisecond timing, virus detection and removal utilities, and software packages for setting up a variety of experiments on the Macintosh. A complete listing of files available can be obtained by using ftp to connect to the archive.

\section{JOINING MACPSYCH}

To join the MacPsych discussion list, send electronic mail to macpsych-request@stolaf.edu, requesting that you be added to the list. This mail will be forwarded to and processed by a real person, so any format and syntax may be used.

When the moderator has received your request, you will be added to the list and you will receive a message explaining the list and introducing you to the archive. Since the list is now sponsored by SCiP and the journal Behavior Research Methods, Instruments, \& Computers (BRMIC), you will also receive an address to which you can write to join SCiP and to subscribe to BRMIC. However, you do not need to be a member of SCiP or subscribe to 
BRMIC to join or to continue as a member of MacPsych. However, both the society and the journal provide valuable information concerning the use of computer technology in research and teaching.

\section{GETTING FILES FROM THE ARCHIVE}

The files on the MacPsych archive are stored on a machine at St. Olaf College and are most easily available by using ftp to connect to that machine. Most of the files are stored in a compressed and translated fashion. This makes it possible for them to be sent over the network to your machine. Thus the process of getting software from MacPsych to your Macintosh requires five steps: (1) logging on to a machine that can connect to the archive by using $\mathrm{ftp}$; (2) using ftp to connect to the archive; (3) transferring the files you want to the machine you are logged into; (4) transferring the files from the machine to your Macintosh; and (5) untranslating and uncompressing the files so your Macintosh can use them.

If your Macintosh is connected directly to a network that is connected to Internet (e.g., you can do ftp and Telnet without logging into another machine), you should consider using Fetch. Fetch enables direct connection to the MacPsych archive and also automatically does the untranslating and uncompressing. It thus reduces the five steps to only two: connecting and downloading. Fetch is currently available on MacPsych.

If you are able to log in to your Internet host directly from your Macintosh you should be able to use the Kermit communication protocol to make the file transfers. Most terminal programs such as Kermit make the process of logging into another computer and transferring files down to your Macintosh fairly simple. Your local computer support center should be able to guide you in the use of the correct terminal program. If you do not have a direct connection to a machine on the Internet, contact your local computer support center for additional help. They can get you an account and get you set up with the appropriate software.

If the machine at your site does not have ftp access to Internet, you will have to receive the files by electronic mail. The section titled Using ftpbymail shows you how to get access to the MacPsych archive if this is your situation. If possible, get an account on a machine that does have ftp access, since $\mathrm{ftp}$ is a much more convenient way to get files from the archive.

\section{Using ftp}

The acronym ftp stands for file transfer protocol. As the name implies, it is a network protocol for transferring files between computers, but it is also the name of a program that implements the protocol and allows you to actually transfer files. It is primarily used by computers that run the UNIX operating system and are connected to high-speed networks like Internet. Anonymous ftp is a form of ftp that does not require you to have an account on the computer you wish to transfer files from.
Any site you wish to use ftp to connect to is uniquely identified by a site name. The site's name is generally several names or acronyms that are separated by periods. In the case of the MacPsych archive, the name of the site is ftp. stolaf.edu. Much like a postal address, the individual components of the name progress from the most specific (ftp) to the most general (edu) description of the site. If the site name does not work for you, send mail to macpsych-request@stolaf.edu and we will attempt to diagnose the problem.

To use ftp, follow the instructions in Appendix A, which presents a sample ftp session with the MacPsych archive. The italicized text below Step 2 is a more detailed explanation of what the step accomplishes. Some of the steps, indicated by an asterisk beside them, are intentionally vague. This is because the way you accomplish this step will vary from site to site. The text in square brackets is nonliteral.

\section{Using ftpbymail}

If the only access you have to MacPsych is via electronic mail, the program ftpbymail will respond to electronic mail queries and will provide, essentially, the functions of $\mathrm{ftp}$. Included here are several examples of using ftpbymail. Appendix B gives the complete syntax of commands for the program. To make a request of ftpbymail, send electronic mail to ftpbymail@stolaf.edu with one or more commands in the body of your message. Your message does not have to have any subject heading. Ftpbymail will respond by carrying out your commands and send you, via electronic mail, the results of its operation. For instance the single line

\section{help}

in the body of a message to ftpbymail@stolaf.edu will result in an electronic mail message's being sent to you that contains instructions about ftpbymail. The following is a sequence of commands that, if included in the body of a message to ftpbymail@stolaf.edu, will result in your receiving electronic mail that lists the contents of the MacPsych directory. The first command is necessary because the archive is located in the directory pub/macpsych.

$$
\begin{aligned}
& \text { cd pub/macpsych } \\
& \text { dir }
\end{aligned}
$$

The following commands will result in your receiving a copy of the file OOREADME.txt:

$$
\begin{aligned}
& \text { cd pub/macpsych } \\
& \text { get 00README.txt }
\end{aligned}
$$

If you request a file that is longer than 1,500 lines, ftpbymail will break the file up into pieces before sending it to you in separate files. You will have to use a local editor to piece the files back together before you untranslate them and uncompress them. Some files on the archive are as large as 3.5 MB. You should check the size of the file you request to make sure you know what to expect in the mail. (The dir command will give you a list of files and their sizes.) 


\section{Untranslating and Uncompressing Files}

Once you have files from MacPsych on your Macintosh, there are two approaches you can use to untranslate and uncompress them. The easiest approach is to use a single program, Stuffit Classic, to accomplish both steps. Stuffit Classic is shareware, and if you use it you should send the $\$ 25$ fee to the company that supports it. A less expensive way involves using two programs, UnStuffit Classic and BinHex. BinHex is the program that will untranslate the file you receive, and Unstuffit will uncompress the file. Both of these programs are freeware and require no fee.

Stuffit Classic, Unstuffit Classic, and BinHex are available on the MacPsych archive. If you do not have a copy of BinHex, you cannot get access to these utilities, since they are stored in compressed and translated form on the archive. To get around this bootstrap problem, a disk containing these utilities will be mailed to anyone who requests them, who also certifies that they cannot obtain them from a local source. Send electronic mail to macpsych-request@ stolaf.edu with a mailing address to which the disk should be sent.

\section{SUBMITTING FILES TO THE ARCHIVE}

\section{Submission Standards}

Because the MacPsych archive is likely to be used by psychologists (and students of psychology) with a range of expertise in things computational, we have established some conventions that will make its use easy and straightforward. In addition, since some of the items archived in MacPsych are based on reports published in various journals (e.g., Behavior Research Methods, Instruments, \& Computers), there needs to be a standard format in which things can be accessed and referenced. Hence the following rules:

1. Materials sent to be archived in MacPsych should be checked for viruses before mailing them to the moderator. To keep up with the continuing trade in viruses, the contributor should be sure to use the most current version of virus detection software. For a free virus detection package, the most current version of Disinfectant is available on MacPsych.

2. Materials sent to be archived in MacPsych should be accompanied by a short abstract, no more than two or three sentences long, describing the contents of the files.

2.1. In addition to the abstract, the following should be noted: the date the items were last updated, the level of support the author will assume, a short reference to published articles describing the items, and (if possible) the contributor's e-mail address. The software (name and version) needed to open and effectively use the item (e.g., More 3.0; Excel 2.1 or higher; Word 3.0b or higher) should also be noted.

2.2. The levels of support currently recognized are as follows: full support (questions probably answered, bug fixes and updates provided, etc.); partial support (easy or intriguing questions may be answered, only catastrophic bugs fixed, updates if you feel like it); minimal support (some mail queries answered); and no support (don't bother asking for anything).

Other levels of support may be negotiated with the moderator. If you do not indicate otherwise, a statement will be added to your abstract stating you will provide no support. We hope these levels will make it possible for people to share their work without feeling any longterm obligation for support.

3. Materials sent to be archived in MacPsych should be accompanied by a ReadMe file, in ASCII or TeachText, describing the contents of the file in more detail. For most items, this file can be compressed along with the other items into one archive file. The ReadMe file should reiterate the information in the short abstract and, in addition, contain at least the following:

3.1. Full references to published articles that describe the item in the archive.

3.2. A statement indicating that you own or have the permission rights to distribute the software. If your software has been described in BRMIC or in other journals, and if your abstract or documentation is drawn from the text of that article, make sure that you have permission from these copyright holders to use the copyrighted text.

3.3. If you wish, a disclaimer releasing you and your institution from liability for the correctness of the software. For suggestions on such a disclaimer, see below.

3.4. A statement indicating known hardware or limiting information regarding the Macintosh operating system. If you have not tested it on a complete set of Macintoshes and operating systems, you should indicate what hardware and operating system you have used (e.g., Mac Plus or Mac IIci with System 6.0.5 and System 7.0.1, respectively).

3.5. In your ReadMe file, and in other documentation, make sure that the first mention of any trademarked item (e.g., Macintosh) has the standard trademark label. Companies may get testy if you do not do this.

4. Materials you send to be archived in MacPsych should be stored in a compressed, BinHexed file. All items except the short abstract should be stored in the same file. The standard compression utility is Stuffit Classic, Version 1.6 (available on MacPsych), but self-expanding archives are also acceptable (such as those made by Compactor). Other formats should be preapproved.

There are additional recommendations for the various kinds of items that may be archived.

For operational software:

If there is more in-depth documentation, it should be in a separate document, preferably in Microsoft Word, MacWrite, or TeachText.

If documentation is included in the program (e.g., through a help facility), this should be mentioned in the abstract.

Notes about incompatibilities and known bugs should be included in the ReadMe file.

The software and any additional documentation should be archived in a single, compressed, BinHexed file. 
For source code files:

There should be in-code explanatory comments.

Notes about incompatibilities and known bugs should be included in the ReadMe document or in in-code comments.

Other standards:

If at all possible, use the recognized standards for other types of items. For instance, graphics in PICT, sounds in snd, and movies in moov.

\section{Submission Procedure}

1. Prepare the items and accompanying documents as mentioned above.

2. Send an electronic mail message to macpsych@stolaf. edu, indicating that you will be making a contribution to the archive.

3. The compressed, BinHexed item can be sent either of two ways. It can be directly uploaded to the directory pub/tmp at the ftp.stolaf.edu site, or it can be sent in an electronic mail message to macpsych@stolaf.edu. Remember, accompanying documents (ReadMe files, documentation, setup files, examples, etc.) should all be compressed into a single file.

4. The moderator will notify you when your item has been archived. Because of the press of duties, the moderator may take a week or more to finish the process.

5 . The moderator may check your files for completeness but cannot guarantee that corruption will not occur at some point in the archiving process. You are encouraged to check the archive yourself to verify that the item you have sent has been archived correctly.

A note on disclaimers. MacPsych will issue the following general disclaimer at the head of the list of abstracts. You will want to use a more specific disclaimer for the particular item you send to MacPsych.

The MacPsych archive is a service provided with the encouragement of the Society for Computers in Psychology (SCiP). In addition, the MacPsych archive serves as the archive site for Macintosh software and files described in the journal Behavior Research Methods, Instruments, \& Computers (BRMIC), SCiP, BRMIC, the operators of MacPsych, and St. Olaf College make no claims concerning the accuracy or correctness of any information or computer code contained herein. Individual files may contain more explicit information about the level of support that can be expected from the author of any software available in the archive. For information about becoming a member of SCiP, please contact Doug Eamon, University of Wisconsin at Whitewater, Whitewater, WI 53190 eamond@ uwwvax.uww.edu. For subscription information about BRMIC, contact the Psychonomic Society at 1710 Fortview Road, Austin, TX 78704.

It is your responsibility to attach your own disclaimer to your item (as an "About ..." menu item, or in the documentation, or ReadMe, etc.). Here is a sample disclaimer you may use or adopt as you wish:

[Item and accompanying items] are provided "as is" without warranty of any kind. [Author and Author's Institution] make no claims concerning the accuracy or correctness of the computer code contained in, or the results of the use of [item and accompanying items]. The entire risk as to the results and performance of [item and accompanying items] is assumed by you. If [item or accompanying items] are defective, you, and not [Author and Author's Institution] assume the entire cost of all necessary servicing, repair, or correction.

\section{FUTURE PLANS}

We hope that the MacPsych archive will become a useful resource for the Psychologists who use the Macintosh in their teaching or research. Readers can help this occur both by subscribing to the discussion list and by either sending useful software to the archive or suggesting useful software and files that might be included. Your contributions and suggestions are welcome.

\section{APPENDIX A \\ An Annotated ftp Session}

1. Log in to your local Internet machine.*

2. At your system prompt type

ftp ftp.stolaf.edu

and hit return. After a brief delay you should see a message like

Connected to ftp.stolaf.edu.

220 nic.stolaf.edu ftp server (Version 5.180 Mon Mar 25 08:15:51 CST 1991) ready

Name (ftp.stolaf.edu:huff):

This creates a connection between your Internet host and the computer that acts as the macpsych fip site. If you get the message "tp.stolaf.edu: unknown host" it means either your site does not recognize fip.stolaf.edu as a legitimate site or there is a problem with the Internet. Quit ftp by typing bye" and check with local system personnel. 
3. Type "anonymous" and hit return. You should see the prompt

331 Guest login ok, send ident as password

Password (ftp.stolaf.edu:anonymous):

4. Type your full login ID and hit return. You should see the message.

230 Guest login ok, access restrictions apply.

$\mathrm{ftp}>$

5. Type "cd pub/macpsych" and hit return. You should see the message

250 CWD command successful.

$\mathrm{ftp}>$

6. Type "ls" and hit return to see a listing of all the files.

7. Decide which file you would like to transfer and type get [filename]

You should see a message like

200 PORT command successful.

150 Opening ASCII mode data connection for [filename] ([nnnn] bytes).

and eventually

226 Transfer complete. local: [filename] remote: [filename] [nnnn] bytes received in [nn] seconds ([n.nn] Kbytes/s).

ftp >

Your file transfer is done, and the file is now in your current local directory (the directory you were in when you typed "ftp"), and it is called whatever follows the "local:" statement above.

8. Type "bye" at the "ftp $>$ " prompt to quit the ftp program and logout from your local Internet host.

*See text for discussion.

\section{APPENDIX B \\ Ftpbymail Syntax}

Ftpbymail understands the simple subset of standard ftp commands listed below. Valid commands can be entered in upper or lower case. When specifying file or directory names, take care to use proper case.

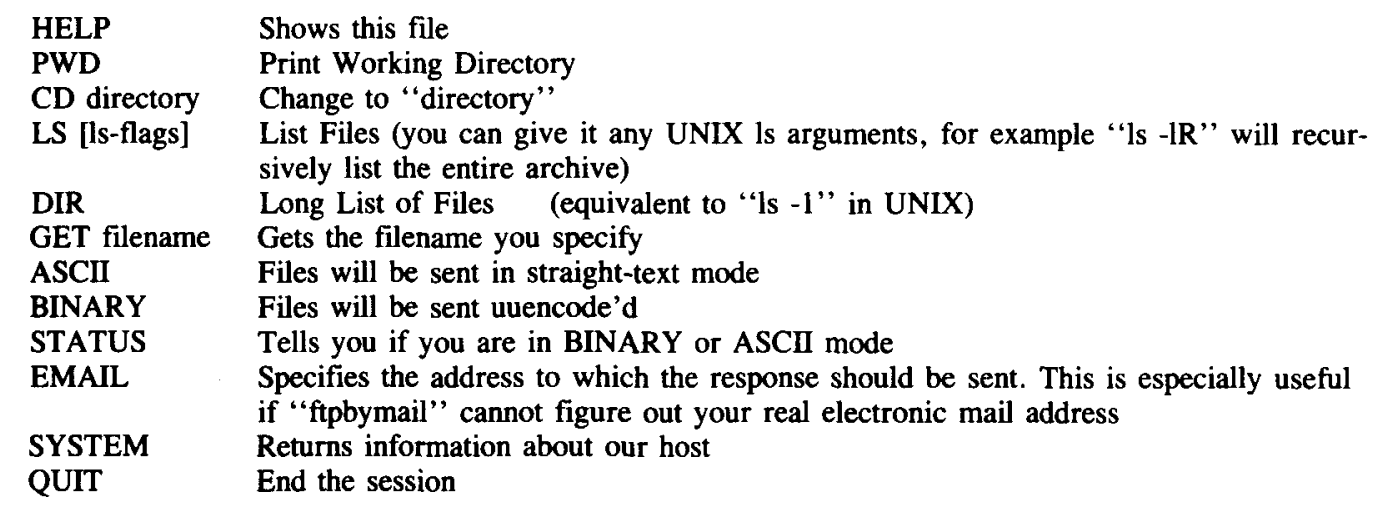

Please email questions to: owner-ftpbymail@stolaf.edu. 